Please do not remove this page

RMIT

UNIVERSITY

\title{
Dihydropyridine inhibition of the glycine receptor: Subunit selectivity and a molecular determinant of inhibition
}

Chen, Xuebin; Cromer, Brett; Webb, Timothy; Yang, Zhe; Hantke, Janina; Harvey, Robert; Parker, Michael https://researchrepository.rmit.edu.au/esploro/outputs/9921858028801341/filesAndLinks?institution=61RMIT_INST\&index=null

Chen, X., Cromer, B., Webb, T., Yang, Z., Hantke, J., Harvey, R., Parker, M., \& Lynch, J. (2009).

Dihydropyridine inhibition of the glycine receptor: Subunit selectivity and a molecular determinant of inhibition. Neuropharmacology, 56(1), 318-327. https://doi.org/10.1016/j.neuropharm.2008.07.001 Document Version: Submitted Version

Published Version: https://doi.org/10.1016/j.neuropharm.2008.07.001

Repository homepage: https://researchrepository.rmit.edu.au

(C) 2008 Elsevier Ltd. All rights reserved.

Downloaded On 2023/04/26 13:40:54 +1000 


\section{DIHYDROPYRIDINE INHIBITION OF THE GLYCINE RECEPTOR: SUBUNIT SELECTIVITY AND A MOLECULAR DETERMINANT OF INHIBITION}

\section{Xuebin Chen ${ }^{1}$, Brett Cromer ${ }^{2}$, Timothy I. Webb ${ }^{1}$, Zhe Yang ${ }^{1}$, Janina Hantke ${ }^{3}$, Robert J. Harvey ${ }^{3}$, Michael W. Parker ${ }^{4}$, and Joseph W. Lynch ${ }^{1}$}

${ }^{1}$ School of Biomedical Sciences and Queensland Brain Institute, University of Queensland, Brisbane, QLD 4072, Australia,

${ }^{2}$ Howard Florey Institute \& Pharmacology Dept., University of Melbourne, Parkville, VIC 3010, Australia.

${ }^{3}$ Department of Pharmacology, The School of Pharmacy, 29-39 Brunswick Square, London WC1N 1AX, UK.

${ }^{4}$ St. Vincent's Institute of Medical Research, Fitzroy, VIC 3065, Australia.

Running title: Dihydropyridine inhibition of glycine receptors

Address correspondence to: Dr. Joseph Lynch, Queensland Brain Institute, University of Queensland, Brisbane, QLD 4072, Australia.

Tel. +617 3346 6375; Fax. +617 3346 6301; Email: j.lynch@uq.edu.au

Number of: Text pages 34, Figures 8, Tables 3, References 32.

Word count: Abstract 250, Introduction 460; Discussion 1316.

Keywords: nifedipine, nicardipine, Cys-loop receptor, chloride channel, inhibitory neurotransmission, binding site. 


\section{Abstract}

The dihydropyridines (DHPs), nifedipine and nicardipine, modulate native glycine receptors (GlyRs) at micromolar concentrations. Nicardipine has a biphasic potentiating and inhibitory effect, whereas nifedipine causes inhibition only. The present study sought to investigate 1) the molecular mechanism by which these compounds inhibit recombinant GlyRs, and 2) their potential utility as subunitselective inhibitors of $\alpha 1, \alpha 1 \beta, \alpha 3$ and $\alpha 3 \beta$ GlyRs. The rate of onset of inhibition in the open state was accelerated by pre-application of DHP in the closed state, with the degree of acceleration proportional to the concentration of pre-applied DHP. This implies a non-inhibitory binding site close to the DHP inhibitory site. DHP inhibition was use-dependent and independent of glycine concentration, consistent with a poreblocking mode of action. DHP sensitivity was abolished by the G2'A mutation, providing a strong case for DHP binding site deep in the pore. Nifedipine exhibited an approximately 10 -fold higher inhibitory potency at $\alpha 1$-containing relative to $\alpha 3$ containing receptors, whereas nicardipine was only weakly selective for $\alpha 1$ containing GlyRs. The differential sensitivities of nifedipine and nicardipine for different GlyR isoforms suggest that DHPs may be a useful resource to screen as pharmacological tools for selectively inhibiting different synaptic GlyR isoforms. 


\section{Introduction}

Glycine receptors (GlyRs) mediate inhibitory neurotransmission in the adult rat spinal cord and brainstem (Lynch, 2004). They incorporate an anion-selective pore and are members of the pentameric Cys-loop family of ligand-gated ion channels. Individual subunits of the Cys-loop receptor family contain a large $\mathrm{N}$-terminal ligandbinding domain followed by a bundle of four transmembrane $\alpha$-helical domains (Unwin, 2005). Each of the five subunits contributes its second transmembrane domain (M2) to the lining of the central water-filled pore. To date, five GlyR subunits have been identified ( $\alpha 1-\alpha 4, \beta)$. Embryonic receptors generally comprise $\alpha 2$ homomers or $\alpha 2 \beta$ heteromers, whereas the dominant adult subtype is the $\alpha 1 \beta$ heteromer (Lynch, 2004). Heteromeric GlyRs are thought to exist in a $2 \alpha: 3 \beta$ stoichiometry (Grudzinska et al., 2005). The $\beta$ subunit, which is widely distributed throughout the adult nervous system, mediates the anchoring of GlyRs to the cytoskeleton via a direct binding interaction with the cytoplasmic protein, gephyrin (Kim et al., 2006). The $\alpha 1-\alpha 4$ subunits exhibit differential central nervous system distribution patterns that are particularly evident in the superficial dorsal horn of the spinal cord (Harvey et al., 2004) and the retina (Haverkamp et al., 2003; Haverkamp et al., 2004; Heinze et al., 2007). The physiological consequences of the differential distribution patterns are difficult to establish as there are currently few pharmacological probes that can selectively inhibit different $\beta$ subunit-containing

GlyR isoforms (Betz and Laube, 2006; Webb and Lynch, 2007). The identification of compounds that can pharmacologically discriminate between $\alpha 1$ - and $\alpha 3$-containing GlyRs may help in understanding the role of $\alpha 3$ GlyRs in inflammatory pain processing in spinal nociceptive neurons (Ahmadi et al., 2002; Harvey et al., 2004; 
Reinold et al., 2005; Zeilhofer, 2005) and in cone signal processing in the retina (Haverkamp et al., 2003).

Dihydropyridines (DHPs) such as nifedipine (NF) and nicardipine (NC) are important for the treatment of hypertension, angina, atrial arrhythmia and myocardial ischaemia (Struyker-Boudier et al., 1990). These drugs act therapeutically by inhibiting calcium flux through L-type $\mathrm{Ca}^{2+}$ channels, thereby inducing the relaxation of vascular smooth muscle and the suppression of cardiac contractility. They inhibit L-type $\mathrm{Ca}^{2+}$ channels at nanomolar concentrations but inhibit other $\mathrm{Ca}^{2+}$ channels at micromolar concentrations (Struyker-Boudier et al., 1990; Dunlap et al., 1995). Low micromolar concentrations of NF and NC have also been shown to modulate native GlyRs in cultured ventral spinal cord neurons (Chesnoy-Marchais and Cathala, 2001). These effects were selective for GlyRs over $\mathrm{GABA}_{\mathrm{A}}$ receptors in the same cells.

In the present study, we examined whether NF and NC may be useful as subunitselective inhibitors of the $\alpha 1, \alpha 1 \beta, \alpha 3$ and $\alpha 3 \beta$ GlyRs. The results suggest that NF may have a reasonable degree of selectivity for $\alpha 1$-containing GlyRs over $\alpha 3$ containing GlyRs. In addition, we have identified a molecular determinant of the inhibitory effect of these compounds. 


\section{Materials and Methods}

\section{Mutagenesis and expression of GlyR cDNAs}

The human GlyR $\alpha 1$, rat $\alpha 3 \mathrm{~L}$ and human $\beta$ subunit $\mathrm{cDNAs}$ were subcloned into the pCIS, pcDNA3.1 and pIRES2-EGFP plasmid vectors, respectively. Site-directed mutagenesis was performed using the QuickChange mutagenesis kit (Stratagene, La Jolla, CA, USA) and the successful incorporation of mutations was confirmed by DNA sequencing. A chimera of the $\alpha 3$ subunit was created whereby the $\alpha 3$ M4 domain residues, A401 - D431, were replaced by the corresponding residues (I393 Q421) of the $\alpha 1$ M4 domain. This chimera was generated according to the "seamless" protocol (Padgett and Sorge, 1996). Briefly, long PCR of the vector encoding $\alpha 3 \mathrm{~L}$ was performed with primers incorporating EarI sites at the termini and this fragment was ligated to a PCR fragment of $\alpha 1$ M4 incorporating compatible EarI sites at each termini. The final construct was confirmed by DNA sequencing.

HEK293 cells, cultured in Dulbecco's Modified Eagles Medium, were transfected using a calcium phosphate precipitation protocol. When co-transfecting GlyR $\alpha$ and $\beta$ subunit constructs, the respective plasmid DNAs were combined in a ratio of 1:10. After exposure to transfection solution for $24 \mathrm{hrs,} \mathrm{cells} \mathrm{were} \mathrm{washed} \mathrm{twice} \mathrm{using}$ calcium-free phosphate buffered saline, then returned to standard culture medium and used for recording over the following $24-72 \mathrm{hrs}$.

\section{Electrophysiology}

The cells were viewed via an inverted fluorescent microscope and currents were recorded in the whole-cell patch-clamp configuration. Cells were superfused by a control solution containing (in $\mathrm{mM}$ ): $140 \mathrm{NaCl}, 5 \mathrm{KCl}, 2 \mathrm{CaCl}_{2}, 1 \mathrm{MgCl}_{2}, 10 \mathrm{HEPES}$, 10 glucose, with the $\mathrm{pH}$ adjusted to 7.4 with $\mathrm{NaOH}$. Patch pipettes were fabricated 
from borosilicate hematocrit tubing (Vitrex, Modulohm, Denmark) and heat polished. Pipettes had a tip resistance of $1-2 \mathrm{M} \Omega$ when filled with the standard pipette solution containing (in mM): $145 \mathrm{CsCl}, 2 \mathrm{CaCl}_{2}, 2 \mathrm{MgCl}_{2}, 10$ HEPES, 10 EGTA, with the $\mathrm{pH}$ adjusted to 7.4 with $\mathrm{NaOH}$. After establishment of the whole-cell configuration, cells were voltage-clamped at $-40 \mathrm{mV}$ (unless otherwise indicated) and membrane currents were recorded using an Axopatch 1D amplifier and pCLAMP9 software (Axon Instruments, Union City, CA, USA). The cells were perfused by a parallel array of microtubular barrels through which solutions were gravity-induced. This system permitted complete solution exchange within $100 \mathrm{~ms}$. Experiments were conducted at room temperature $\left(19-22^{\circ} \mathrm{C}\right)$.

Because $\alpha$ homomers can form functional GlyRs, it is necessary to confirm the incorporation of $\beta$ subunits into functional $\alpha \beta$ heteromers. As the GlyR $\beta$ subunit cDNA was cloned into the pIRES2-EGFP plasmid vector, we used GFP fluorescence to identify cells expressing the GlyR $\beta$ subunit. The successful incorporation of $\beta$ subunits into functional receptors was also inferred by their characteristic reduction in picrotoxin (PTX) sensitivity (Pribilla et al., 1992; Handford et al., 1996) as demonstrated in Fig. 1.

PTX, picrotoxinin (PTXININ), NF and NC were all obtained from Sigma (St Louis, MO, USA) and stored frozen as $50 \mathrm{mM}$ stocks in dimethylsulfoxide.

\section{Data Analysis}

Results are expressed as mean \pm standard error of the mean of three or more independent experiments. The Hill equation was used to calculate the half-maximal concentration $\left(\mathrm{EC}_{50}\right)$ and Hill coefficient $\left(\mathrm{n}_{\mathrm{H}}\right)$ values for glycine activation. A similar equation was also used to calculate the half maximal concentrations for inhibition $\left(\mathrm{IC}_{50}\right)$ and $\mathrm{n}_{\mathrm{H}}$ values of the antagonists tested in this study. All curves were fitted 
using a non-linear least squares algorithm (Sigmaplot 9.0, Jandel Scientific, San Rafael, CA, USA). Statistical significance was determined by paired or unpaired Student's t-test, as appropriate, with $\mathrm{P}<0.05$ representing significance. One-way ANOVA (GraphPad Prism, Version 4 for Windows, GraphPad Software, Inc., San Diego, CA, USA) was used for the comparison of means of more than three groups. 


\section{Results}

\section{Expression and functional properties of GlyR isoforms}

Examples of the response of cells expressing $\alpha 3$ and $\alpha 3 \beta$ GlyRs to increasing concentrations of glycine are shown in Fig. 1A. Glycine concentration-responses, averaged from at least three cells expressing the $\alpha 1, \alpha 1 \beta, \alpha 3$ and $\alpha 3 \beta$ GlyRs, are presented in Fig. 1B with the mean $\mathrm{EC}_{50}$ and $\mathrm{n}_{\mathrm{H}}$ values of best fit presented in Table 1. This table also includes the averaged $\mathrm{EC}_{50}$ and $\mathrm{n}_{\mathrm{H}}$ values for all mutant GlyRs examined in this study. The $\mathrm{EC}_{50}$ values for the $\alpha 3$ and $\alpha 3 \beta$ GlyRs are several fold higher than previously reported by others (Meier et al., 2005; Miller et al., 2005). The reasons for this are unknown. However, similar results are consistently observed in our laboratory (Yang et al., 2007). The incorporation of $\beta$ subunits into functional GlyRs results in a characteristic reduction in receptor sensitivity to PTX (Pribilla et al., 1992; Handford et al., 1996). To confirm the successful incorporation of $\beta$ subunits into functional GlyRs, the effects of $10 \mu \mathrm{M}$ PTX on currents activated by an $\mathrm{EC}_{50}(30 \mu \mathrm{M})$ glycine concentration were compared at the $\alpha 1$ and $\alpha 1 \beta$ GlyRs. The averaged results confirm the expected reduction in PTX sensitivity in the $\alpha 1 \beta$ GlyR (Fig. 1D). We have recently demonstrated that PTXININ is better than PTX at pharmacologically discriminating between $\alpha 3$ homomeric and $\alpha 3 \beta$ heteromeric GlyRs (Yang et al., 2007). We therefore compared the inhibitory potency of $1 \mu \mathrm{M}$ PTXININ on currents activated by $\mathrm{EC}_{50}(300 \mu \mathrm{M})$ glycine at the $\alpha 3$ and $\alpha 3 \beta$ GlyRs (e.g., Fig. 1C). The percentage of original current remaining in $1 \mu \mathrm{M}$ PTXININ, averaged from four cells expressing each receptor, is presented in Fig. 1D. These results confirm that co-expression of $\alpha 1$ or $\alpha 3$ together with the $\beta$ subunit results in the formation of heteromeric receptors. 


\section{Mechanism of DHP action}

As described below (Fig. 5), NF and NC exhibited no direct effect in the absence of glycine. In addition to its inhibitory effect, $\mathrm{NC}$ has also been reported to potentiate currents activated by sub-saturating glycine concentrations (Chesnoy-Marchais and Cathala, 2001). We also found that NC exerts a dual potentiating and inhibitory effect on $\alpha 1$ and $\alpha 1 \beta$ GlyRs. For homomeric $\alpha 1$ GlyRs, potentiation was observed only at glycine concentrations less than $\mathrm{EC}_{30}$. However, in many cells the $\mathrm{NC}$ potentiating response was labile, either disappearing completely or progressively declining in magnitude over time (data not shown). NC produced only inhibition of $\alpha 3$ and $\alpha 3 \beta$ GlyRs, regardless of the glycine concentration used (e.g., Fig. 6A, below). The onset of potentiation of $\alpha 1$ GlyRs by NC was faster than that of inhibition, as shown by the biphasic response to increasing concentrations of $\mathrm{NC}$ in the presence of $\mathrm{EC}_{20}(20 \mu \mathrm{M})$ glycine (Fig. 2A, upper panel). The potentiating concentration-response, averaged from 11 cells (Fig. 2B), was bell-shaped with a peak at around $30 \mu \mathrm{M}$, possibly due to truncation of the potentiation peak by a faster onset of inhibition at higher NC concentrations. Examples of inhibition by $\mathrm{NC}$ of currents activated by $\mathrm{EC}_{50}(30 \mu \mathrm{M})$ glycine in homomeric $\alpha 1$ GlyRs are shown in Fig. 2A (lower panel), with averaged concentration-responses presented in Fig. 2B. The averaged $\mathrm{IC}_{50}$ and $\mathrm{n}_{\mathrm{H}}$ values for NC inhibition are given in Table 2. Examples of the inhibitory effects of NF on currents activated by $\mathrm{EC}_{50}$ glycine at the $\alpha 1$ GlyR are shown in Fig. $2 \mathrm{C}$ and the averaged inhibitory concentration-response is plotted in Fig. 2D. The averaged parameters of best fit to the inhibitory concentration-response are given in Table 2 .

We next investigated whether DHP inhibition was dependent of glycine concentration. In this experiment, the inhibitory potencies of NF $(10 \mu \mathrm{M})$ and $\mathrm{NC}(30$ $\mu \mathrm{M})$ were compared at $\mathrm{EC}_{20}, \mathrm{EC}_{50}$ and saturating glycine concentrations on 
homomeric $\alpha 1$ and $\alpha 3$ GlyRs, respectively. As indicated in Fig. 3A, inhibition by both $\mathrm{NF}$ and $\mathrm{NC}$ is glycine-independent.

To investigate voltage-dependence, we activated homomeric $\alpha 1$ GlyRs with an $\mathrm{EC}_{50}$ glycine concentration and compared the percentage inhibition induced by NF $(10 \mu \mathrm{M})$ or $\mathrm{NC}(30 \mu \mathrm{M})$ at $-80,-20$ and $+40 \mathrm{mV}$. The pooled results, summarised in Fig. 3B, reveal that the inhibition by both compounds is not significantly voltagedependent. Such a result is expected given that both compounds are uncharged.

While the above experiments quantified the effects of extracellularly applied DHPs, we also sought to determine whether NF could access a binding site from the intracellular membrane surface. With $100 \mu \mathrm{M}$ NF in the patch pipette, we found that the magnitude of whole-cell $\alpha 1$ GlyR current did not change significantly during the first 10 min of whole-cell recording ( $\mathrm{n}=3$ cells, data not shown), and that currents with magnitudes $>10 \mathrm{nA}$ were invariably observed. We therefore conclude that DHPs access their site from the extracellular membrane surface only. This agrees with the results of a similar experiment performed on native spinal neuronal GlyRs (ChesnoyMarchais and Cathala, 2001).

We then investigated the use-dependence of NF and NC at homomeric $\alpha 1$ GlyRs as shown in Fig. 4A. First, a saturating $(2 \mathrm{mM})$ glycine concentration was applied to cells, then the time course of onset of the inhibitory response to $10 \mu \mathrm{M} N \mathrm{NF}$ was determined (Fig. 4A, left panel). NF was then pre-applied for $10 \mathrm{~s}$ in the absence of glycine, then glycine plus NF were co-applied (second panel). The large magnitude of the initial glycine-induced current spike indicates that NF applied in the closed state does not inhibit GlyRs as efficiently as when applied in the open state, indicating that the inhibition is use-dependent. Surprisingly, however, the rate of onset of NF inhibition in the open state was accelerated by the pre-application of NF in the closed state. We next pre-applied NF in the closed state, and then simultaneously removed 
NF while applying glycine (Fig. 4A, third panel). Following activation of the channels, the onset of the NF inhibitory response followed exactly the same time course as when glycine was applied in the continued presence of NF, as in panel 2. However, as expected in the absence of NF, this inhibition was not sustained and the recovery from inhibition occurred at around the same rate as seen in the first panel. This result strongly suggests that NF is able to associate closely with the GlyR in the closed state without efficiently inhibiting the current, implying the existence of a separate site to which NF can bind in the closed state without producing inhibition. It is only when the GlyR is activated that NF can reach its inhibitory binding site. We term these sites the 'non-inhibiting' and 'inhibiting' sites, respectively. Panel 4 in Fig. 4A is a control showing that the simultaneous co-application of glycine plus NF produces a slower onset of inhibition similar to that seen in panel 1. To show this more clearly, the time course of the onset of inhibition by NF under each of the four experimental conditions (labelled $1-4$ in Fig. 4A) are shown normalised on an expanded time scale in Fig. 4B. These curves were adequately fit with single exponentials (not shown) and the respective time constants averaged from three cells subject to the same experimental procedure are summarised in Fig. 4C. A similar pattern of activity was observed with $\mathrm{NC}$ as summarised in Fig. 4D.

To investigate the functional properties of the non-inhibiting site, we performed two sets of experiments that are summarised in Fig. 5. In an attempt to quantitate the NF sensitivity of the non-inhibiting site, we applied increasing concentrations of NF for $30 \mathrm{~s}$ periods and determined the time constant of inhibition onset when $2 \mathrm{mM}$ glycine was subsequently applied. A sample experiment, which also included regular control glycine applications, is shown in Fig. 5A. The time courses of the onset of current inhibition under each of the indicated NF concentrations (labelled 1-5) are shown normalised on an expanded time scale in Fig. 5B. This panel also shows that 
30 and $60 \mathrm{~s}$ applications of $0.3 \mu \mathrm{M}$ NF have no measurably different effect on the rate or magnitude of inhibition, thus indicating that NF had equilibrated with a noninhibiting binding site within $30 \mathrm{~s}$ under the conditions employed in this experiment. The curves in Fig. 5B were adequately fit with single exponentials and the time constants averaged from four cells are summarised in Fig. 5C. When plotted against concentration on a semi-log plot, the time constants were well fitted by a linear regression. These data clearly show that the rate of onset of the inhibition is dependent on the concentration of pre-applied NF. As discussed in detail below, this result is not consistent with a single non-inhibiting DHP site per receptor. It is more consistent with a 'reservoir' that can accommodate a number of DHP molecules in proportion to the free external DHP concentration.

Fig. 5D plots the averaged peak currents (triangles) and averaged minimum currents (circles) as a function of NF concentration. The averaged NF inhibitory concentration-response measured in the presence of glycine (reproduced from Fig. 2D) is included as a dashed line for comparison. That the circles provide a surprisingly good fit to the dashed line indicates that a given concentration of NF applied in the closed state causes the same degree of inhibition as when the same concentration of NF is applied in the open state. In other words, the NF molecules in free solution and NF molecules that bind to the non-inhibiting site both seem to create a similar NF concentration at the inhibiting site when the channels are opened. Rather less can be inferred from the concentration-dependence of peak current magnitude (triangles in Fig. 5D). The decline in peak current magnitude seen at higher NF concentrations is a consequence of the faster onset of inhibition relative to the rate of channel opening which is limited by the solution exchange rate.

We next sought to determine the reversibility of NF binding at the non-inhibiting site and to compare the NF dissociation rates from the non-inhibiting and inhibiting 
sites. To address these questions, we applied a $10 \mu \mathrm{M}$ concentration of NF for $30 \mathrm{~s}$, then waited a varying period of time $(0,2,5$ and $30 \mathrm{~s})$ before adding $2 \mathrm{mM}$ glycine. A sample experiment, which includes regular glycine-only controls, is shown in Fig 5E. The time courses of inhibition onset in response to each of the indicated NF concentrations (labelled I - IV) are shown normalised on an expanded time scale in Fig. 5F. Two controls (both labelled V) recorded in the absence of a prior NF application are also included but are superimposed in Fig. 5F. Time constants of best fit to these curves, averaged from three cells, are summarised in Fig. 5G. The same results are also shown normalised to the maximum time constants recorded in the same cell (Fig. 5H). It is apparent that the half recovery time lies between 5 and $30 \mathrm{~s}$. By interpolating between these values using sigmoidal curve fits to the data from each cell, we estimated a mean half recovery time of $9.7 \pm 2.2 \mathrm{~s}(\mathrm{n}=3)$. This result provides a measure of the time course with which NF unbinds from the non-inhibiting site in the closed state. The mean half recovery time from the inhibiting site, measured directly from the $0 \mathrm{~s}$ trace in the same three cells, was $7.9 \pm 1.1 \mathrm{~s}(\mathrm{n}=3)$. These values are not significantly different, possibly suggesting that NF dissociation from the noninhibiting site controls the rate at which it dissociates from the inhibiting site as well.

\section{Subunit selectivity}

The steady-state inhibitory concentration-responses of NC and NF at the $\alpha 1, \alpha 3$, $\alpha 1 \beta$ and $\alpha 3 \beta$ GlyRs were measured in the presence of an $\mathrm{EC}_{50}$ glycine concentration. The glycine concentrations used in these experiments are listed in Table 2. Sample experiments are displayed in Fig. 6A. The averaged concentration-responses for NF at each of the four GlyR subtypes are displayed in Fig. 6B. All averaged $\mathrm{IC}_{50}$ and $\mathrm{n}_{\mathrm{H}}$ values of best fit are presented in Table 2 . The results indicate that homomeric $\alpha 1$ GlyRs are significantly more sensitive than homomeric $\alpha 3$ GlyRs to inhibition by NF. 
They also show that incorporation of the $\beta$ subunit produces an increased NF

sensitivity. This increase was not statistically significant for heteromeric $\alpha 1 \beta$ GlyRs, but was significant $(\mathrm{P}<0.05$, Student's unpaired t-test) for $\alpha 3 \beta$ GlyRs. A similar pattern was seen with NC. The averaged concentration-responses for NC at each of the four GlyR subtypes are displayed in Fig. 6C, with parameters of best fit summarised in Table 2. These results suggest that NF could be useful for pharmacologically discriminating $\alpha 1$ - from $\alpha 3$-containing GlyRs.

\section{Molecular determinants of DHP inhibition}

As use-dependence and non-competitive inhibition are characteristic of open channel blockers, we investigated whether DHPs bind in the pore. As indicated in the M2 domain sequence alignment (Fig. 7A), the pore-lining domains of the $\alpha 1$ and $\alpha 3$ subunits differ only in the identity of the 2' pore-lining residue, whereas the $\beta$ subunit displays a low sequence homology throughout most of its length. Non-conserved residues at the 2' and 6' positions are thought to provide the binding site for PTX (Zhorov and Bregestovski, 2000; Shan et al., 2001; Yang et al., 2007) and ginkgolides (Kondratskaya et al., 2005; Hawthorne et al., 2006; Heads et al., 2008) and possibly also to cyanotriphenylborate (Rundstrom et al., 1994) and butyrolactones (Steinbach et al., 2000). Indeed, a DHP binding site at the 6' position was predicted on the basis of a structural comparison of the GlyR pore with the L-type calcium channel DHP binding site (Chesnoy-Marchais and Cathala, 2001). Given these considerations, we investigated the possibility that DHPs might also bind in the 2' -6 ' region and that the differential sensitivity of NF for $\alpha 1$ and $\alpha 3$ GlyRs might be explained by the nonconserved residue at the ${ }^{2}$ ' position. We further hypothesised that the effect of the $\beta$ subunit on NF sensitivity may be mediated by the amino acid differences at the 2 ' and/or 6' positions. 
We therefore measured the NF and NC inhibitory concentration-responses at the $\alpha 1^{\mathrm{G} 2^{\prime} \mathrm{A}}, \alpha 1^{\mathrm{G} 2{ }^{\prime} \mathrm{P}}$ and $\alpha 1^{\mathrm{G} 2^{\prime} \mathrm{A}} \beta$ GlyRs. Due to the low sensitivities of the $\alpha 1^{\mathrm{G} 2}$ 'A and $\alpha 1^{\mathrm{G} 2}$ 'P GlyRs to both NF and NC, it was not possible to generate full inhibitory concentration-response curves. However, it was evident that the $\mathrm{IC}_{50}$ values for both compounds were $>100 \mu \mathrm{M}$ (Table 2). Fig. 7B shows the averaged inhibitory concentration-responses for NF and NC at the $\alpha 1 \beta, \alpha 3 \beta$ and $\alpha 1^{\mathrm{G} 2}{ }^{\prime} \mathrm{A} \beta$ GlyRs, with the mean parameters of best fit summarised in Table 2 . The low NF sensitivity of the $\alpha 3 \beta$ GlyR can be transferred to the $\alpha 1 \beta$ GlyR via the $\alpha 1$ subunit G2'A mutation. Thus, the 2' residue appears to be a specific determinant of DHP sensitivity. However, because the homomeric $\alpha 1^{\mathrm{G} 2 \text { 'A }}$ GlyR has a lower DHP sensitivity than either the homomeric $\alpha 1$ or $\alpha 3$ GlyRs (Table 2), it is likely that other differences between the $\alpha 3$ and $\alpha 1$ GlyR also contribute to DHP sensitivity. The sensitivity of homomeric $\alpha 1$ GlyRs to $\mathrm{NC}$ is also abolished by the $\alpha 1^{\mathrm{G} 2}$ 'A mutation (Fig. 7B, Table 2).

The role of the $\alpha 1$ subunit 6' residue in mediating the subunit-specific effects of $\mathrm{NF}$ and $\mathrm{NC}$ was investigated by measuring $\mathrm{IC}_{50}$ values at the $\alpha 1^{\mathrm{T} 6}{ }^{\prime} \mathrm{F}$ GlyR (the $\alpha$ to $\beta$ subunit substitution) and at the more conservative $\alpha 1^{\mathrm{T} 6^{\prime} \mathrm{A}}$ and $\alpha 1^{\mathrm{T} 6^{\prime} \mathrm{S}}$ GlyRs. As shown in Fig. 7C and summarised in Table 2, the averaged $\mathrm{NF}$ and $\mathrm{NC} \mathrm{IC}_{50}$ values were not significantly changed by any of these mutations. Threonine residues can participate in hydrogen bonds via their hydroxyl groups and hydrophobic interactions via their methyl groups. To investigate more specifically possible hydrogen bonding with 6 ' threonines, we also examined the effect of the T6'V mutation, which replaces the $\mathrm{OH}$ with a methyl group. As this mutation also had no significant effect on receptor sensitivity to either NF or NC (Fig 7C and Table 2), we conclude that 6' threonines are not important for interactions with DHPs, by either hydrogen bonds or hydrophobic interactions. 
As discussed below, our functional data (as summarised in Figs. 4 and 5) suggest that DHP may bind to a site in or near the external surface of the lipid bilayer. In an attempt to identify this site, we took advantage of the fact that $\alpha 1$ and $\alpha 3$ subunits are differentially sensitive to NF and hypothesised that non-conserved residues in the transmembrane or adjacent extracellular domains may mediate this differential sensitivity. With the exception of M4 and the C-terminal tail, the amino acid sequences of the $\alpha 1$ and $\alpha 3$ subunits are highly conserved throughout all transmembrane and adjacent external domains. A sequence alignment of the M4 and C terminal tail (Fig. 8) reveals a total of ten non-conserved residues, many of which are reactive. The $\mathrm{C}$-terminal tail of the $\alpha 3$ subunit also contains two additional residues relative to the $\alpha 1$ subunit. To determine whether this region mediates the differential sensitivity to NF, we made a chimera of $\alpha 3$ and $\alpha 1$ subunits (termed $\alpha 3^{{ }^{-1}}$ ${ }^{\mathrm{M} 4}$ ) where residues A401 - D431 of the $\alpha 3$ M4 domain were replaced by the corresponding residues $\mathrm{I} 393-\mathrm{Q} 421$ of the $\alpha 1$ subunit. We also mutated to alanines each of the $\alpha 1$ residues shown as shaded in Fig. 8, and deleted the final two residues of the $\alpha 3$ subunit. The tested constructs were as follows: $\alpha 1^{1393 \mathrm{~A} / \mathrm{G} 394 \mathrm{~A}}$ (double mutant), $\alpha 1^{\mathrm{M} 397 \mathrm{~A}}, \alpha 1^{\mathrm{M} 404 \mathrm{~A}}, \alpha 1^{\mathrm{R} 415 \mathrm{~A}}, \alpha 1^{\mathrm{H} 419 \mathrm{~A}}$ (which is conserved but reactive),

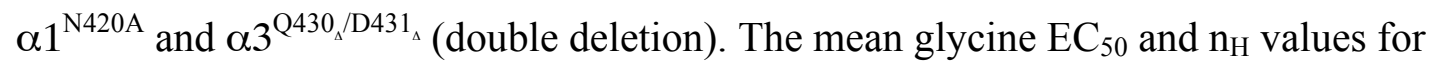
all these constructs are summarised in Table 1. Each construct was investigated by quantitating the percentage inhibition produced by $10 \mu \mathrm{M}$ NF of currents activated by the respective $\mathrm{EC}_{50}$ glycine concentration as given in Table 3. As summarised in Table 3, the $\alpha 3^{{ }^{\alpha 1}}{ }^{-\mathrm{M} 4}$ and $\alpha 3$ GlyRs exhibited similar NF sensitivities, thus eliminating this domain as a determinant of the differential NF sensitivity. Furthermore, the results obtained using the other M4 mutant $\alpha 1$ GlyRs provide no evidence that nonconserved M4 residues form the NF binding site. An interesting outcome of these 
experiments is that the high glycine sensitivity of the $\alpha 3^{{ }^{\alpha 1}}{ }^{-M} 4$ chimera implies a role for the M4 domain in GlyR gating. 


\section{Discussion}

\section{Location of the inhibitory site}

An unusual feature is that while pre-incubation with DHP does not directly inhibit glycine-activated currents, DHP pre-incubation increases the subsequent rate of inhibition. This indicates the presence of both inhibiting and non-inhibiting sites, with only the non-inhibiting site being accessible in the closed state. Binding to the noninhibiting site enhances access to the inhibiting site when channels are opened. This discussion will consider the locations of both sites, starting with the inhibiting site.

Our data do not differentiate between desensitisation, allosteric inhibition or direct block as possible mechanisms of DHP inhibition. However, two lines of evidence strongly suggest that DHPs bind in the channel pore. First, functional data indicate that DHP inhibition is use-dependent and independent of glycine concentration, both classical characteristics of open channel blockers. Second, the relatively conservative $\alpha 1^{\mathrm{G} 2} \mathrm{~A}$ mutation caused a dramatic loss in DHP inhibitory potency, suggesting this mutation ablated a pore binding site. However, the $\alpha 3$ GlyR, which shares an identical M2 domain amino acid sequence with the $\alpha 1^{\mathrm{G} 2}$ 'A GlyR, retained weak sensitivity to DHP inhibition suggesting that other domains of the GlyR also contribute to the DHP sensitivity differences between $\alpha 1$ and $\alpha 3$ GlyRs.

The original hypothesis proposed that DHPs were coordinated by 6' threonines (Chesnoy-Marchais and Cathala, 2001). However, our results indicate that DHP inhibition is insensitive to 6' mutations. Threonines can contribute to both hydrogen bonds and hydrophobic interactions. However, selective elimination of their hydrophobic interaction capability by the T6'S mutation, and of their hydrogen bonding capability by the T6'V mutation, and of both binding capabilities by the 
T6'A mutation, had minimal effects on DHP inhibitory potency. These results are incompatible with a direct binding interaction between DHPs and 6' Thr residues.

Molecular docking studies, performed as described in Hawthorne et al. (2006), established the feasibility of DHP block of GlyRs at the 2' level in the pore (not shown). However, due to uncertainties associated with the homology model and our poor understanding of the nature of conformational changes associated with channel activation, this model cannot be considered reliable predictor of the specific details of binding.

Based on all these considerations, we conclude that DHPs most likely bind at the 2' pore-lining position, where they are coordinated primarily by backbone groups near the 2' level.

\section{The non-inhibiting site}

Let us first consider the possibility of a single non-inhibiting DHP site per receptor. In this scenario, the number of channels that have DHP bound at a single non-inhibiting site will increase with concentration, and this will be reflected by an increased magnitude of inhibition when the channels are opened. However, the rate of DHP transition from the non-inhibiting site to the inhibiting site should be concentration-independent as the required proximity of the two sites would demand that the effective local concentration be either very high or zero. This model is inconsistent with our data because the rate of DHP inhibition depends on the concentration of DHP at the non-inhibiting site.

Our results are more consistent with a non-inhibiting 'site' or 'reservoir' that can simultaneously accommodate multiple DHP molecules with an occupancy rate proportional to the DHP concentration in the external solution. Such a model would 
explain our observation (Fig. 6B and C) that the rate of DHP inhibition is proportional to the DHP concentration in the external solution.

The cell membrane is one candidate for this site as DHPs are known to partition into the lipid bilayer and concentrate at the lipid-water interface (Herbette et al., 1989; Mason et al., 1992; Herbette et al., 1994). However, for a direct transition to occur between the non-inhibiting and inhibiting sites, they need to be closely located. It is difficult to conceive how DHPs at the external lipid-water interface could more efficiently access an inhibitory site located deep in the pore than DHPs in free aqueous solution. Alternatively a DHP inhibitory site could lie between the TM regions, accessible from the protein-lipid interface and be allosterically affected by the G2'A mutation. There is however, no direct evidence for such a site, as inhibition by NF was unaffected by mutations at the $15^{\prime}$ position or to non-conserved lipidexposed TM4 residues in the alpha1 and alpha3 subunits.

The remaining possibility is that multiple DHP molecules may bind simultaneously in a region of the pore that is external to but close to the 2' inhibitory site. It has recently been proposed that hydrophobic pore binding molecules of comparable size to NF (e.g., PTX) can bind in crevices between M2 domains in the $\mathrm{GABA}_{\mathrm{A}}$ receptor (Law and Lightstone, 2008). Theoretically, such a mechanism could permit five DHPs molecules to bind in each GlyR pore without producing block. We believe our results are most plausibly described by a model whereby DHP molecules bind in this configuration in the closed state. Channel opening would release these molecules into the pore where they bind to the higher affinity 2 ' inhibitory site. The challenge is to demonstrate that DHP molecules can bind in this location in a noninhibiting configuration. 
Inhibition of $\alpha 3$-containing GlyRs by the inflammatory mediator, $\mathrm{PGE}_{2}$, in spinal cord dorsal horn results in the disinhibition of firing in nociceptive projection neurons (Ahmadi et al., 2002; Harvey et al., 2004). This mechanism may underlie inflammation-induced hyperalgesia or allodynia (Zeilhofer, 2005; Lynch and Callister, 2006). Immunohistochemical and electrophysiological evidence indicates that $\alpha 3 \beta$ GlyRs are incorporated into synapses on these neurons and that approximately $50 \%$ of $\alpha 3$ subunit containing-synapses in dorsal horn neurons also costain for the $\alpha 1$ subunit (Harvey et al., 2004). It is not known why the $\alpha 3$ subunit is strongly expressed in these neurons, but is sparsely distributed throughout the remainder of the spinal cord. Investigation of the kinetic properties, sub-cellular distributions and relative contributions to the net glycinergic synaptic current by $\alpha 1-$ and $\alpha 3$-containing GlyRs may help explain their presence and could provide important insights into pain processing mechanisms in the spinal cord. As NF inhibits $\alpha 1 \beta$ GlyRs at a 12 -fold greater potency than it inhibits $\alpha 3 \beta$ GlyRs, it may be useful as a tool for selectively isolating $\alpha 3 \beta$ glycinergic mini-synaptic currents in voltage clamp recordings. However, as NF may inhibit voltage-gated $\mathrm{K}^{+}$and $\mathrm{Ca}^{2+}$ channels (Fagni et al., 1994; Dunlap et al., 1995), it may not be useful in investigating the roles of $\alpha 1$ - and $\alpha 3$-mediated inhibitory synaptic currents on neuronal firing properties. Our findings also suggest that the enormous array of DHP analogues already generated in attempt to find better therapies for cardiovascular disorders may provide a useful resource to screen for even more selective pharmacological tools.

\section{Conclusions}

We propose that the inhibitory GlyR DHP binding site is located near the 2' position in the GlyR pore. The precise location of the non-inhibitory DHP site is less certain. Because this site must be able to coordinate multiple DHP molecules 
simultaneously and must also lie close to the inhibitory site, we suggest the most likely explanation is that DHP molecules bind in between adjacent M2 domain helices, five per receptor, without inhibiting current flux. As NF is a moderately selective inhibitor of $\alpha 1 \beta$ relative to $\alpha 3 \beta$ GlyRs, it may be useful as a pharmacological tool for investigating the role of synaptic GlyR isoforms in pain processing mechanisms in spinal nociceptive neurons and cone signal processing in the retina. Moreover, the differential sensitivity of NF and NC suggests that DHPs may be a useful compound class to screen for novel GlyR inhibitors with improved subunit-selectivity. 


\section{Acknowledgements}

This study was supported by project grants from the National Health and Medical Research Council of Australia to JWL and to MWP and BAC. MWP and JWL are both supported by National Health and Medical Research Council Research Fellowships. BAC is supported by a Sir Randal Heymanson Fellowship. RJH and JH are supported by grants from the Medical Research Council (G0500833) and the Heptagon Fund. We are grateful to Ms Justine Haddrill and Agnieszka Ney for expert assistance with site-directed mutagenesis. 


\section{References}

Ahmadi, S., Lippross, S., Neuhuber, W. L., Zeilhofer, H. U. 2002. PGE(2) selectively blocks inhibitory glycinergic neurotransmission onto rat superficial dorsal horn neurons. Nat Neurosci 5, 34-40.

Betz, H., Laube, B. 2006. Glycine receptors: recent insights into their structural organization and functional diversity. J Neurochem 97, 1600-1610.

Chesnoy-Marchais, D., Cathala, L. 2001. Modulation of glycine responses by dihydropyridines and verapamil in rat spinal neurons. Eur J Neurosci 13, 2195-2204.

Dunlap, K., Luebke, J. I., Turner, T. J. 1995. Excocytotic calcium channels in mammalian central neurons. Trends Neurosci 18, 89-98.

Fagni, L., Bossu, J. L., Bockaert, J. 1994. Inhibitory effects of dihydropyridines on macroscopic $\mathrm{K}+$ currents and on the large conductance $\mathrm{Ca}(2+)$-activated $\mathrm{K}+$ channel in cultured cerebellar granule cells. Pflugers Arch 429, 176-182.

Grudzinska, J., Schemm, R., Haeger, S., Nicke, A., Schmalzing, G., Betz, H., Laube, B. 2005. The beta subunit determines the ligand binding properties of synaptic glycine receptors. Neuron 45, 727-739.

Handford, C. A., Lynch, J. W., Baker, E., Webb, G. C., Ford, J. H., Sutherland, G. R., Schofield, P. R. 1996. The human glycine receptor beta subunit: primary structure, functional characterisation and chromosomal localisation of the human and murine genes. Brain Res Mol Brain Res 35, 211-219.

Harvey, R. J., Depner, U. B., Wassle, H., Ahmadi, S., Heindl, C., Reinold, H., Smart, T. G., Harvey, K., Schutz, B., Abo-Salem, O. M., Zimmer, A., Poisbeau, P., Welzl, H., Wolfer, D. P., Betz, H., Zeilhofer, H. U., Muller, U. 2004. GlyR alpha3: an essential target for spinal PGE2-mediated inflammatory pain sensitization. Science 304, 884-887.

Haverkamp, S., Muller, U., Harvey, K., Harvey, R. J., Betz, H., Wassle, H. 2003. Diversity of glycine receptors in the mouse retina: localization of the alpha3 subunit. J Comp Neurol 465, 524-539.

Haverkamp, S., Muller, U., Zeilhofer, H. U., Harvey, R. J., Wassle, H. 2004. Diversity of glycine receptors in the mouse retina: localization of the alpha2 subunit. J Comp Neurol 477, 399-411.

Hawthorne, R., Cromer, B. A., Ng, H. L., Parker, M. W., Lynch, J. W. 2006. Molecular determinants of ginkgolide binding in the glycine receptor pore. J. Neurochem. 98, 395-407.

Heads, J. A., Hawthorne, R. L., Lynagh, T., Lynch, J. W. 2008. Structure-activity analysis of ginkgolide binding in the glyicne receptor pore. J Neurochem In press.

Heinze, L., Harvey, R. J., Haverkamp, S., Wassle, H. 2007. Diversity of glycine receptors in the mouse retina: localization of the alpha4 subunit. J Comp Neurol 500, 693-707.

Herbette, L. G., Mason, P. E., Sweeney, K. R., Trumbore, M. W., Mason, R. P. 1994. Favorable amphiphilicity of nimodipine facilitates its interactions with brain membranes. Neuropharmacology 33, 241-249.

Herbette, L. G., Vant Erve, Y. M., Rhodes, D. G. 1989. Interaction of 1,4 dihydropyridine calcium channel antagonists with biological membranes: lipid bilayer partitioning could occur before drug binding to receptors. J Mol Cell Cardiol 21, 187-201. 
Kim, E. Y., Schrader, N., Smolinsky, B., Bedet, C., Vannier, C., Schwarz, G., Schindelin, H. 2006. Deciphering the structural framework of glycine receptor anchoring by gephyrin. Embo J 25, 1385-1395.

Kondratskaya, E. L., Betz, H., Krishtal, O. A., Laube, B. 2005. The beta subunit increases the ginkgolide B sensitivity of inhibitory glycine receptors. Neuropharmacology 49, 945-951.

Law, R. J., Lightstone, F. C. 2008. Gaba receptor insecticide non-competitive antagonists may bind at allosteric modulator sites. Int J Neurosci 118, 705734.

Lynch, J. W. 2004. Molecular structure and function of the glycine receptor chloride channel. Physiol Rev 84, 1051-1095.

Lynch, J. W., Callister, R. J. 2006. Glycine receptors: a new therapeutic target in pain pathways. Curr Opin Investig Drugs 7, 48-53.

Mason, R. P., Moisey, D. M., Shajenko, L. 1992. Cholesterol alters the binding of $\mathrm{Ca} 2+$ channel blockers to the membrane lipid bilayer. Mol. Pharmacol. 41, 315-321.

Meier, J. C., Henneberger, C., Melnick, I., Racca, C., Harvey, R. J., Heinemann, U., Schmieden, V., Grantyn, R. 2005. RNA editing produces glycine receptor alpha3(P185L), resulting in high agonist potency. Nat Neurosci 8, 736-744.

Miller, P. S., Da Silva, H. M., Smart, T. G. 2005. Molecular basis for zinc potentiation at strychnine-sensitive glycine receptors. J Biol Chem 280, 37877-37884.

Padgett, K. A., Sorge, J. A. 1996. Creating seamless junctions independent of restriction sites in PCR cloning. Gene 168, 31-35.

Pribilla, I., Takagi, T., Langosch, D., Bormann, J., Betz, H. 1992. The atypical M2 segment of the beta subunit confers picrotoxinin resistance to inhibitory glycine receptor channels. Embo J 11, 4305-4311.

Reinold, H., Ahmadi, S., Depner, U. B., Layh, B., Heindl, C., Hamza, M., Pahl, A., Brune, K., Narumiya, S., Muller, U., Zeilhofer, H. U. 2005. Spinal inflammatory hyperalgesia is mediated by prostaglandin $\mathrm{E}$ receptors of the EP2 subtype. J Clin Invest 115, 673-679.

Rundstrom, N., Schmieden, V., Betz, H., Bormann, J., Langosch, D. 1994. Cyanotriphenylborate: subtype-specific blocker of glycine receptor chloride channels. Proc Natl Acad Sci U S A 91, 8950-8954.

Shan, Q., Haddrill, J. L., Lynch, J. W. 2001. A single beta subunit M2 domain residue controls the picrotoxin sensitivity of alphabeta heteromeric glycine receptor chloride channels. J Neurochem 76, 1109-1120.

Steinbach, J. H., Bracamontes, J., Yu, L., Zhang, P., Covey, D. F. 2000. Subunitspecific action of an anticonvulsant thiobutyrolactone on recombinant glycine receptors involves a residue in the M2 membrane-spanning region. Mol Pharmacol 58, 11-17.

Struyker-Boudier, H. A., Smits, J. F., De Mey, J. G. 1990. The pharmacology of calcium antagonists: a review. J Cardiovasc Pharmacol 15, S1-10.

Unwin, N. 2005. Refined structure of the nicotinic acetylcholine receptor at 4A resolution. J Mol Biol 346, 967-989.

Webb, T. I., Lynch, J. W. 2007. Molecular pharmacology of the glycine receptor chloride channel. Curr Pharm Des 13, 2350-2367.

Yang, Z., Cromer, B. A., Harvey, R. J., Parker, M. W., Lynch, J. W. 2007. A proposed structural basis for picrotoxinin and picrotin binding in the glycine receptor pore. J Neurochem 103, 580-589.

Zeilhofer, H. U. 2005. The glycinergic control of spinal pain processing. Cell Mol Life Sci 62, 2027-2035. 
Zhorov, B. S., Bregestovski, P. D. 2000. Chloride channels of glycine and GABA receptors with blockers: Monte Carlo minimization and structure-activity relationships. Biophys J 78, 1786-1803. 


\section{Figure legends}

Fig. 1. Glycine and PTX sensitivities of $\alpha 1, \alpha 1 \beta, \alpha 3$ and $\alpha 3 \beta$ GlyRs. A. Examples of currents activated by the indicated glycine concentrations in cells expressing $\alpha 3$ and $\alpha 3 \beta$ GlyRs. In this and all subsequent figures, inward currents are represented as downward deflections and unfilled bars represent the period of glycine application. The listed glycine concentrations apply to both sets of traces. B. Averaged concentration-responses for the $\alpha 1, \alpha 1 \beta, \alpha 3$ and $\alpha 3 \beta$ GlyRs. Mean parameters of best fit are given in Table 1. C. Examples of the inhibitory effects of $1 \mu \mathrm{M}$ PTXININ on currents activated by $\mathrm{EC}_{50}$ glycine in cells expressing $\alpha 3$ and $\alpha 3 \beta$ GlyRs. PTXININ was applied for the period indicated by the filled bar. Displayed traces were recorded from the same two cells as shown in A. D. Averaged inhibition produced by $10 \mu \mathrm{M}$ PTX or $1 \mu \mathrm{M}$ PTXININ at each of the four indicated GlyR subtypes. A Student's unpaired t-test was used to assess statistical significance, with ${ }^{*} \mathrm{P}<0.05,{ }^{* *} \mathrm{P}<0.01$, ${ }^{* * *} \mathrm{P}<0.001$ representing significance relative to the corresponding homomeric GlyR.

Fig. 2. Concentration-dependent effects of NC and NF at homomeric $\alpha 1$ subunit GlyRs. A. The left panel shows examples of the effects of increasing concentrations of NC on currents activated by $\mathrm{EC}_{20}(20 \mu \mathrm{M})$ and $\mathrm{EC}_{50}(30 \mu \mathrm{M})$ glycine in different cells. Note that potentiation is seen only on $\mathrm{EC}_{20}$ responses. The right panel shows the potentiating concentration-response (unfilled circles) averaged from 11 cells recorded at the $\mathrm{EC}_{20}$ glycine concentration. The inhibitory concentration-response, averaged from four cells at $\mathrm{EC}_{50}$ glycine, is shown as filled squares. Parameters of best fit to the inhibitory concentration-response are given in Table 2 . No attempt was made to fit the potentiating concentration-response. B. The left panel shows examples of the effects of increasing concentrations of NF on currents activated by $\mathrm{EC}_{50}(30 \mu \mathrm{M})$ 
glycine. The right panel shows the concentration-response averaged from four cells. Parameters of best fit to the Hill equation are given in Table 2.

Fig. 3. Glycine concentration-dependence and voltage-dependence of DHP inhibition at homomeric $\alpha 1$ subunit GlyRs. A. Effects of $10 \mu \mathrm{M}$ NF and $30 \mu \mathrm{M}$ NC on currents activated by $\mathrm{EC}_{20}(20 \mu \mathrm{M}), \mathrm{EC}_{50}(30 \mu \mathrm{M})$ and $\mathrm{EC}_{100}(2 \mathrm{mM})$ glycine averaged from five cells. There was no significant glycine-dependence for either compound. B. Averaged percentage inhibition induced by $10 \mu \mathrm{M}$ NF and $30 \mu \mathrm{M}$ NC on currents activated by $\mathrm{EC}_{50}$ glycine at $-80,-20$ and $+40 \mathrm{mV}$. Results represent the average of five cells. There was no significant voltage-dependence for either compound.

Fig. 4. Use-dependence of DHP inhibition at homomeric $\alpha 1$ subunit GlyRs. A. NF was applied at a concentration of $10 \mu \mathrm{M}$ and glycine was applied at $2 \mathrm{mM}$ using four different experimental protocols as indicated. All displayed traces were recorded from the same cell. The horizontal lines represent the closed state (the upper-most line), the NF-inibited state and the open state (lower-most line), respectively. B. The onset of the NF inhibitory response under all four experimental conditions shown in A (labelled 1 - 4) are shown normalised, expanded and superimposed. Traces 1 and 2 are displayed as thick lines and traces 3 and 4 as thin lines. C. Exponential fits to the onset of the NF inhibitory response, averaged from three cells, for each of the four experimental conditions shown in A. D. Similar experiment to $\mathrm{C}$ using $30 \mu \mathrm{M} \mathrm{NC}$ in place of NF.

Fig. 5. Characterisation of the 'non-inhibiting' binding site on homomeric $\alpha 1$ GlyRs. A. Examples of the effects of $30 \mathrm{~s}$ applications of $0.3,1,10$ and $30 \mu \mathrm{M} N F$ in the closed state. The effect of a $60 \mathrm{~s}$ application of $0.3 \mu \mathrm{M}$ NF is also shown. A $2 \mathrm{mM}$ 
concentration of glycine was used to activate the channels to observe the subsequent response. Regular $2 \mathrm{mM}$ glycine controls are also applied. B. The onset of the NF inhibitory response for each experimental condition shown in A (labelled 1-5) are shown normalised, expanded and superimposed. Trace 2 is displayed as a dashed line. C. Time constants fitted to the onset of the NF inhibitory response, averaged from four cells, as a function of NF concentration. D. Averaged peak currents (triangles) and minimum currents (circles) seen upon application of glycine after a $30 \mathrm{~s}$ preapplication of the indicated NF concentration in the closed state. The minimum currents (circles) were normalised to current magnitudes measured at the same time point in the control traces, to take account of desensitisation. The NF inhibitory concentration-response curve, reproduced from Fig. 3D, is included for comparison. E. Examples of the effects of $30 \mathrm{~s}$ applications of $30 \mu \mathrm{M} N F$ in the closed state, followed by a varying 'washout' period $(0,2,5$ and $30 \mathrm{~s})$ in control solution before the application of $2 \mathrm{mM}$ glycine. Regular $2 \mathrm{mM}$ glycine controls are also applied. F. The onset of the NF inhibitory response for each experimental condition shown in A (labelled I - V) are shown normalised, expanded and superimposed. The two control traces labelled V are displayed as dashed lines, although traces IV and V overlap. G. Time constants fitted to the onset of the NF inhibitory response, averaged from three cells, plotted as a function of the NF washout time. The column labelled 'no NF' corresponds to trace $\mathrm{V}$ which was recorded in the absence of NF pre-exposure. $\mathrm{H}$. This shows the same data as in G normalised to the maximum (no NF) time constant in each cell. It shows that the mean NF half recovery time was between 5 and $30 \mathrm{~s}$ at the non-inhibiting site.

Fig. 6. Sensitivity of $\alpha 1, \alpha 1 \beta, \alpha 3$, and $\alpha 3 \beta$ GlyRs to inhibition by NF and NC. A. Examples of the effects of $10 \mu \mathrm{M} \mathrm{NF}$ and $30 \mu \mathrm{M} \mathrm{NC}$ on $\mathrm{EC}_{50}$ glycine currents in $\alpha 1$ 
and $\alpha 3$ GlyRs. We selected an $\alpha 1$-expressing cell in which the NC potentiating response had run down. B. Averaged concentration-response curves for NF at each of the four receptor subtypes. B. Averaged concentration-response curves for NC at each of the four receptor subtypes. All averaged $\mathrm{IC}_{50}$ and $\mathrm{n}_{\mathrm{H}}$ values are presented in Table 2.

Fig. 7. Investigation into the molecular determinants of DHP inhibition. A. Sequence alignment of the $\alpha 1, \alpha 3$ and $\beta$ subunit M2 domains. Non-conserved residues at the pore-lining 2' and 6' positions are shown in bold. B. Averaged concentrationresponses for $\mathrm{NF}$ and $\mathrm{NC}$ at the $\alpha 1^{\mathrm{G} 2}{ }^{\prime} \mathrm{A} \beta$ GlyR. Parameters of best fit are displayed in Table 2 . The curve fits for the respective $\alpha 1 \beta$ and $\alpha 3 \beta$ concentration-responses are included for comparison. C. The left and right panels show the averaged concentration-responses for NF and NC, respectively, at the $\alpha 1^{\mathrm{T} 6^{\prime} \mathrm{A}}, \alpha 1^{\mathrm{T} 6^{\prime} \mathrm{S}}, \alpha 1^{\mathrm{T} 6^{\prime} \mathrm{F}}$ and $\alpha 1^{\mathrm{T}{ }^{\prime} \mathrm{V}}$ GlyRs. Parameters of best fit are displayed in Table 2. The inhibitory concentration-response curve fits for NF and NC at the $\alpha 1$ GlyR are included for comparison.

Fig. 8. Sequence alignment of the M4 and C-terminal tail regions of the $\alpha 1$ and $\alpha 3$ subunits. Non-conserved residues are shown in bold. Residues mutated in this study are highlighted in grey. The dashed line represents the M4 $\alpha$-helical region with bold dashes representing the membrane embedded portion, according to the model of Unwin (Unwin, 2005). The intracellular and extracellular limits of the M4 $\alpha$-helix are denoted as 'in' and 'out', respectively. K389 was not tested as it was considered too distant from the extracellular membrane surface to represent a likely DHP binding site. 
Table 1. Glycine activation properties of all GlyRs employed in this study.

\begin{tabular}{|c|c|c|c|}
\hline GlyR & $\mathrm{EC}_{50}$ & $n_{\mathrm{H}}$ & $n$ \\
\hline$\alpha 1$ & $30 \pm 2$ & $1.7 \pm 0.2$ & 5 \\
\hline$\alpha 1 \beta$ & $21 \pm 1$ & $2.0 \pm 0.1$ & 4 \\
\hline$\alpha 3$ & $309 \pm 19 * * *$ & $2.0 \pm 0.1$ & 6 \\
\hline$\alpha 3 \beta$ & $246 \pm 13^{* * *}$ & $1.6 \pm 0.1$ & 3 \\
\hline$\alpha 1^{\mathrm{G} 2 ’ \mathrm{~A}}$ & $22 \pm 3$ & $1.9 \pm 0.3$ & 5 \\
\hline$\alpha 1^{\mathrm{G} 2 ' \mathrm{~A}} \beta$ & $19 \pm 5$ & $1.7 \pm 0.1$ & 3 \\
\hline$\alpha 1^{\mathrm{G} 2 ’ \mathrm{P}}$ & $147 \pm 27 * *$ & $2.5 \pm 0.3$ & 5 \\
\hline$\alpha 1^{\mathrm{T} 6^{\prime} \mathrm{A}}$ & $1.4 \pm 0.3 * * *$ & $1.1 \pm 0.2$ & 8 \\
\hline$\alpha 1^{\mathrm{T} 6^{\prime} \mathrm{S}}$ & $1.1 \pm 0.2^{* * *}$ & $1.1 \pm 0.3$ & 4 \\
\hline$\alpha 1^{\mathrm{T} 6^{\prime} \mathrm{F}}$ & $6.4 \pm 1.1 * * *$ & $1.5 \pm 0.1$ & 6 \\
\hline$\alpha 1^{\mathrm{T} 6^{\prime} \mathrm{V}}$ & $610 \pm 200^{*}$ & $0.8 \pm 0.1$ & 4 \\
\hline$\alpha 1^{\mathrm{I} 393 \mathrm{~A} / \mathrm{G} 394 \mathrm{~A}}$ & $61 \pm 6^{* *}$ & $2.5 \pm 0.2$ & 6 \\
\hline$\alpha 1^{\mathrm{M} 397 \mathrm{~A}}$ & $37 \pm 6$ & $2.3 \pm 0.3$ & 4 \\
\hline$\alpha 1^{\mathrm{M} 404 \mathrm{~A}}$ & $38 \pm 6$ & $2.2 \pm 0.3$ & 4 \\
\hline$\alpha 1^{\mathrm{R} 415 \mathrm{~A}}$ & $49 \pm 8$ & $2.0 \pm 0.1$ & 6 \\
\hline$\alpha 1^{\mathrm{H} 419 \mathrm{~A}}$ & $144 \pm 25^{* *}$ & $1.7 \pm 0.1$ & 5 \\
\hline$\alpha 1^{\mathrm{N} 420 \mathrm{~A}}$ & $96 \pm 8^{* * *}$ & $2.4 \pm 0.1$ & 3 \\
\hline$\alpha 3^{\mathrm{Q} 430_{\Delta} / \mathrm{D} 431_{\Delta}}$ & $381 \pm 20 * * * \#$ & $1.7 \pm 0.2$ & 4 \\
\hline$\alpha 3^{a^{1-M 4}}$ & $123 \pm 13 * * * \# \# \#$ & $1.4 \pm 0.1$ & 5 \\
\hline
\end{tabular}

${ }^{*} \mathrm{P}<0.05,{ }^{* *} \mathrm{P}<0.01$ and ${ }^{* * *} \mathrm{P}<0.001$ by unpaired Student's t-test relative to $\alpha 1$ GlyR. $\# \mathrm{P}<0.05$ and \#\#\#P<0.001 by unpaired Student's t-test relative to $\alpha 3$ GlyR.

$\alpha 3^{\mathrm{N} 30_{\Delta} / \mathrm{D} 431_{\Delta}}$ refers to the $\alpha 3$ GlyR with the last two residues of $\mathrm{C}$-terminus removed $\alpha 3^{{ }^{1-M} 4}$ refers to the $\alpha 3$ GlyR with its M4 and C-terminal domains replaced by the corresponding part of $\alpha 1$ GlyR. 
Table 2. $\mathrm{IC}_{50}$ and $\mathrm{n}_{\mathrm{H}}$ values for $\mathrm{NF}$ and $\mathrm{NC}$ at wild type and mutant GlyRs

\begin{tabular}{|c|c|c|c|c|c|c|c|}
\hline \multirow[b]{2}{*}{ GlyR } & \multirow[b]{2}{*}{$\begin{array}{c}{[\text { Glycine }]} \\
(\mu \mathrm{M})\end{array}$} & \multicolumn{3}{|c|}{ NF } & \multicolumn{3}{|c|}{$\mathrm{NC}$} \\
\hline & & $\mathrm{IC}_{50}(\mu \mathrm{M})$ & $\mathrm{n}_{\mathrm{H}}$ & $\mathrm{n}$ & $\mathrm{IC}_{50}(\mu \mathrm{M})$ & $\mathrm{n}_{\mathrm{H}}$ & $\mathrm{n}$ \\
\hline$\alpha 1$ & 30 & $3.3 \pm 1.5$ & $0.54 \pm 0.04$ & 5 & $10.6 \pm 6.0$ & $0.70 \pm 0.11$ & 4 \\
\hline$\alpha 1 \beta$ & 30 & $1.2 \pm 0.7$ & $0.54 \pm 0.12$ & 4 & $5.8 \pm 2.3$ & $0.77 \pm 0.08$ & 5 \\
\hline$\alpha 3$ & 300 & $\begin{array}{l}29.2 \pm \\
3.3 * * *\end{array}$ & $0.79 \pm 0.11$ & 4 & $30.0 \pm 9.5$ & $1.07 \pm 0.17$ & 5 \\
\hline$\alpha 3 \beta$ & 300 & $13.7 \pm 1.9 * *$ & $0.65 \pm 0.08$ & 3 & $11.4 \pm 2.2$ & $0.66 \pm 0.06$ & 3 \\
\hline$\alpha 1^{\mathrm{G} 2} \mathrm{~A}$ & 30 & $>100$ & & 5 & $>100$ & & 5 \\
\hline$\alpha 1^{\mathrm{G} 2^{\prime} \mathrm{P}}$ & 150 & $>100$ & & 5 & $>100$ & & 5 \\
\hline$\alpha 1^{\mathrm{G} 2^{\prime} \mathrm{A}} \beta$ & 30 & $32.0 \pm 7.2 * *$ & $1.09 \pm 0.15$ & 3 & $24.0 \pm 2.8$ & $0.75 \pm 0.05$ & 6 \\
\hline$\alpha 1^{\mathrm{T} 6 \mathrm{~A}}$ & 1 & $3.9 \pm 1.5$ & $0.62 \pm 0.04$ & 4 & $3.0 \pm 0.9$ & $1.25 \pm 0.21$ & 4 \\
\hline$\alpha 1^{\text {T6'S }}$ & 1 & $5.6 \pm 2.6$ & $0.74 \pm 0.10$ & 5 & $8.7 \pm 2.2$ & $0.78 \pm 0.12$ & 5 \\
\hline$\alpha 1^{\mathrm{T} \sigma^{\prime} \mathrm{F}}$ & 5 & $4.5 \pm 1.6$ & $0.52 \pm 0.05$ & 6 & $15.4 \pm 6.2$ & $0.50 \pm 0.03$ & 3 \\
\hline$\alpha 1^{\mathrm{T} 6^{\prime} \mathrm{V}}$ & 600 & $1.4 \pm 0.3$ & $0.70 \pm 0.13$ & 4 & $9.2 \pm 1.8$ & $1.02 \pm 0.11$ & 4 \\
\hline
\end{tabular}

[Glycine] refers to the $\mathrm{EC}_{50}$ glycine concentration used to activate the currents in order to measure NF and NC inhibitory concentration-responses.

Asterisks represent significance relative to $\alpha 1$ GlyR values by unpaired Student's ttest. $* \mathrm{P}<0.05, * * \mathrm{P}<0.01, * * * \mathrm{P}<0.001$ 
Table 3. NF sensitivity of wild type and M4 mutant GlyRs

\begin{tabular}{cccc}
\hline GlyR & $\begin{array}{c}{[\text { Glycine }]} \\
\mathrm{EC}_{50}(\mu \mathrm{M})\end{array}$ & $\begin{array}{c}\text { \% current remaining in the } \\
\text { presence of } 10 \mu \mathrm{M} \text { NF }\end{array}$ & $\mathrm{n}$ \\
\hline$\alpha 1$ & 30 & $33.9 \pm 8.7$ & 4 \\
$\alpha 3$ & 300 & $68.2 \pm 1.9^{* *}$ & 4 \\
$\alpha 1^{\mathrm{I393A} / \mathrm{G} 394 \mathrm{~A}}$ & 60 & $28.5 \pm 7.8$ & 4 \\
$\alpha 1^{\mathrm{M} 397 \mathrm{~A}}$ & 40 & $24.6 \pm 6.1$ & 4 \\
$\alpha 1^{\mathrm{M} 404 \mathrm{~A}}$ & 40 & $18.5 \pm 5.9$ & 5 \\
$\alpha 1^{\mathrm{R} 415 \mathrm{~A}}$ & 40 & $25.4 \pm 4.8$ & 4 \\
$\alpha 1^{\mathrm{H} 419 \mathrm{~A}}$ & 130 & $20.8 \pm 3.6$ & 3 \\
$\alpha 1^{\mathrm{N} 420 \mathrm{~A}}$ & 90 & $16.2 \pm 2.2$ & 4 \\
$\alpha 3^{\mathrm{N} 430 / \mathrm{D} 431_{\Delta}}$ & 350 & $55.3 \pm 6.0$ & 3 \\
$\alpha 3^{{ }^{1-\mathrm{M} 4}}$ & 120 & $74.1 \pm 5.7 *$ & 3
\end{tabular}

${ }^{*} \mathrm{P}<0.05$ and $* * \mathrm{P}<0.01$ by unpaired Student's t-test relative to $\alpha 1$ GlyR.

[Glycine] refers to the $\mathrm{EC}_{50}$ glycine concentration used to activate the currents in order to measure NF inhibitory potency. 